\section{Pré-natal de gestantes de risco habitual por enfermeira obstetra e obstetriz: custo- -efetividade sob a perspectiva do Sistema de Saúde Suplementar}

\author{
Prenatal care for normal-risk pregnant women by \\ obstetric nurses and midwives: cost-effectiveness \\ from the perspective of the Supplementary \\ Health System in Brazil

\section{Período prenatal de gestantes de riesgo habitual con enfermera obstetra y partera: costo- efectividad desde la perspectiva del Sistema de Salud Suplementario}

Mariane de Oliveira Menezes 1

Roxana Knobel 2

Carla Betina Andreucci 3

Claudia Garcia Magalhães 1

Melania Maria Ramos Amorim 4

Leila Katz 4

Maíra Libertad Soligo Takemoto 1

\section{Resumo}

Em diversos países, a atenção primária às gestantes é conduzida por enfermeiras obstetras elou obstetrizes. No Sistema Suplementar de Saúde no Brasil, a cobertura da assistência pré-natal é obrigatória e realizada por médicos obstetras. O objetivo deste estudo é conduzir análise de custo-efetividade, comparando desfechos clínicos e custos associados à incorporação do pré-natal por enfermeiras obstetras e obstetrizes no âmbito do Sistema de Saúde Suplementar, sob a perspectiva da operadora de planos de saúde como fonte pagadora. Foi construída uma árvore de decisão, baseada nos dados de metanálise da Colaboração Cochrane que mostrou redução do risco de parto prematuro no grupo de gestantes de risco habitual acompanhado por enfermeiras obstetras e obstetrizes. Foram considerados apenas os custos médicos diretos cobertos pelas operadoras de planos de saúde para a realização de consultas e exames essenciais, conforme protocolo do Ministério da Saúde vigente. Assumiu-se custo unitário de consulta com cada profissional como iguais e aplicou-se um aumento do custo global com exames pré-natais associado ao acompanhamento médico, conforme dado obtido na literatura. Estimou-se a razão de custo-efetividade incremental de $-R \$ 10.038,43$ (economia de $R \$ 10.038,43$ ) por parto prematuro evitado. Esse resultado mostrou-se consistente nas análises de sensibilidade, com economias associadas à substituição variando de $-R \$$ 2.544, 60 até $-R \$ 31.807,46$ por parto prematuro evitado. Como conclusão, observou-se que o cuidado pré-natal por enfermeiras obstetras e obstetrizes é superior ao prestado por médicos obstetras para o desfecho prevenção de parto prematuro, resultando ainda em economia de recursos.

Gasto em Saúde; Análise Custo-Benefício; Cuidado Pré-Natal; Obstetrícia; Promoção da Saúde

\author{
Correspondência \\ M. O. Menezes \\ Universidade Estadual Paulista. \\ Av. Prof. Mário Rubens Guimarães Montenegro s/n, Botucatu, \\ SP 18618-687, Brasil. \\ mariane.menezes@unesp.br \\ 1 Universidade Estadual Paulista, Botucatu, Brasil.
2 Universidade Federal de Santa Catarina, Florianópolis, Brasil.
3 Universidade Federal de São Carlos, São Carlos, Brasil.
4 Instituto de Medicina Integral Prof. Fernando Figueira, Recife,
Brasil.
}




\section{Introdução}

Em muitos países do mundo a atenção primária às gestantes é conduzida por enfermeiras obstetras e/ou obstetrizes, sendo exemplos a Austrália, o Canadá e o Reino Unido, entre outros 1. Em todo o mundo, diversos modelos de atenção coexistem em cada contexto - desde cuidado centrado inteiramente nessas profissionais até esquemas de cuidado misto, em conjunto com médicos de família ou obstetras 1 . No contexto brasileiro, a regulamentação da atuação de enfermeiras obstetras e/ou obstetrizes é feita pela lei do exercício profissional de enfermagem (Lei no 7.498/19862) que dispõe que estes profissionais são os titulares do diploma de graduação de Enfermeira associado ao diploma de especialização em Enfermagem Obstétrica ou diploma de graduação de Obstetriz, além de estabelecer suas atribuições.

A Confederação Internacional de Obstetrizes (ICM) estabelece internacionalmente as competências essenciais para a prática de obstetrícia por enfermeiras obstetras e/ou obstetrizes, incluindo desde o atendimento rotineiro de pré-natal até a identificação de complicações com necessidade de encaminhamento, além de atividades de educação em saúde perinatal ${ }^{3}$. Em 2016, a Organização Mundial da Saúde (OMS) divulgou recomendações sobre cuidados pré-natais para uma experiência positiva na gravidez, elencando um leque ampliado de ações e estratégias, incluindo múltiplos atores, dentre eles as enfermeiras obstetras e/ou obstetrizes. O documento recomenda ainda a continuidade dos cuidados prestados por esses profissionais, em que uma parteira ou grupo de parteiras conhecidas presta cuidado desde o pré-natal até o pós-parto 4. Dois anos depois, em 2018, a OMS publicou recomendações a respeito de intervenções não clínicas para reduzir cesarianas desnecessárias, dentre as quais destaca-se a atuação multiprofissional com a participação das enfermeiras obstetras e/ou obstetrizes no cuidado à gestante e parturiente 5 .

Anualmente, ocorrem cerca de 500 mil nascimentos em serviços de maternidade vinculados ao Sistema de Saúde Suplementar no Brasil (Departamento de Informática do SUS. Nascidos vivos Brasil. http://tabnet.datasus.gov.br/cgi/tabcgi.exe?sinasc/cnv/nvuf.def, acessado em 29/Abr/2019). Segundo dados do inquérito nacional Nascer no Brasil, mais de $80 \%$ desses nascimentos ocorrem por cirurgias cesarianas, com sérias repercussões para a saúde materno-infantil no Brasil, no curto, médio e longo prazos 6,7,8,9. Ainda conforme dados do estudo Nascer no Brasil, observa-se no país uma significativa ampliação do acesso à assistência pré-natal, atingindo a cobertura pré-natal universal em alguns contextos, contudo, ainda com baixa adequação, tanto pelo início tardio quanto por um número abaixo do indicado de consultas e déficit na realização dos exames de rotina, orientações sobre o parto e aleitamento 10. Outro dado relevante evidenciando pelo mesmo estudo 11 e corroborado por outros pesquisadores 12,13 é a elevada taxa brasileira de nascimentos prematuros, que foi quase o dobro da encontrada em países europeus. Para o período 2011-2012, a taxa foi estimada em 11,5\%, sendo que nascimentos pré-termo que foram iniciados pelo profissional de saúde (não espontâneos) foram $39,3 \%$ do total. Desse montante, a maioria deve-se predominantemente (90\%) às cesáreas realizadas fora de trabalho de parto, em particular em serviços vinculados ao Sistema de Saúde Suplementar 11.

No Sistema de Saúde Suplementar brasileiro está prevista atualmente a cobertura obrigatória da assistência pré-natal e exames necessários pelos planos de saúde com Obstetrícia, contudo, eles são realizados integralmente por médicos, em sua maioria médicos especialistas (médicos de família de família ou obstetras). Apesar de haver cobertura garantida para o acompanhamento pré-natal por médico, as evidências científicas disponíveis 1,14 indicam diferença significativa entre os dois tipos de acompanhamento (por médico obstetras e por enfermeiras obstetras e/ou obstetrizes). Essa diferenciação acontece tanto no que diz respeito ao conteúdo do acompanhamento quanto aos desfechos associados, não sendo adequado considerar os dois tipos de acompanhamento como sendo iguais ou intercambiáveis. Além das evidências científicas representadas por revisões sistemáticas com metanálise de ensaios clínicos randomizados (GRADE alto), também diretrizes nacionais e internacionais 3,4,5,15,16,17 recomendam a inserção das enfermeiras obstetras e/ou obstetrizes no contexto do pré-natal. Desse modo, o objetivo deste estudo é conduzir avaliação econômica de custo-efetividade para comparar desfechos clínicos e custos associados à incorporação do acompanhamento pré-natal realizado por enfermeiras obstetras e/ou obstetrizes no âmbito do Sistema de Saúde Suplementar, adotando a perspectiva da operadora de planos de saúde como fonte pagadora. 


\section{Métodos}

Para subsidiar a construção de um modelo de decisão para conduzir a avaliação econômica, foram utilizados os resultados de uma revisão sistemática com metanálise conduzida por pesquisadores da Colaboração Cochrane 1, que contêm evidências comparativas de dois modelos de oferta de cuidados pré-natais para gestantes de risco habitual. A população, intervenção, comparadores e desfechos apresentados nessa revisão foram considerados coincidentes com os objetivos da presente avaliação de custo-efetividade. Os resultados da revisão sistemática com metanálise de Sandall et al. 1 indicaram que há uma redução consistente e estatisticamente significativa do risco de parto prematuro $(<37$ semanas) no grupo de gestantes de baixo risco que acompanham pré-natal com enfermeiras obstetras e/ou obstetrizes, quando comparado ao grupo acompanhado por médicos de família ou obstetras. As autoras observaram um risco relativo (RR) de 0,71 (intervalo de 95\% de confiança [IC95\%]: 0,54; 0,92), representando uma redução de $29 \%$ no risco de parto prematuro, com base em ensaio clínico randomizado de alta qualidade (GRADE Alto). Adicionalmente, a análise dos desfechos de segurança indica que não parece haver riscos incrementais associados ao modelo de cuidados pré-natais prestados por enfermeiras obstetras e/ou obstetrizes, sendo que não houve diferença para hospitalização na gestação, taxa de cesárea, complicações maternas na gestação ou parto e desfechos adversos perinatais. Esses achados são corroborados também pelos resultados de outra revisão sistemática com objetivos similares conduzida por Villar et al. 14.

Baseando-se nesses dados, definiu-se pela condução de avaliação econômica do tipo custo-efetividade, que pressupõe que haja uma diferença de eficácia entre os comparadores 18. Para isso, foi construído um modelo do tipo árvore de decisão 18, baseado nos dados de eficácia obtidos de Sandall et al. 1. Adotou-se como medida de resultado a razão de custo-efetividade incremental (RCEI), que é calculada como a razão (divisão) entre a diferença de custos (custo da nova alternativa menos o custo da tecnologia atualmente disponível) e a diferença de efetividades (efetividade da nova alternativa menos a efetividade da tecnologia atualmente disponível). Consideramos, para esta análise, o desfecho de taxa de partos prematuros, de modo que os custos incrementais da nova tecnologia (pré-natal com enfermeiras obstetras e/ou obstetrizes) foram ponderados pela redução no número de partos prematuros, de modo que a RCEI foi expressa como custo por parto prematuro evitado.

O diagrama da árvore de decisão utilizada esta apresentado na Figura 1. A população elegível considerada no modelo foram as gestantes de risco habitual, atendidas em serviços vinculados ao Sistema de Saúde Suplementar. Os comparadores considerados foram: (i) acompanhamento pré-natal com consultas regulares com enfermeiras obstetras e/ou obstetrizes e (ii) acompanhamento pré-natal com consultas regulares com médico de família ou obstetras. A perspectiva adotada na análise foi a da operadora de plano de saúde como fonte pagadora (Planserv Bahia. Valores referenciais. http:// www.planserv.ba.gov.br/prestador/valores-referenciais/; Tabelas Planserv. http://www.planserv. ba.gov.br/prestador/tabelas-planserv/, acessado em 30/Abr/2019). Foram considerados apenas os custos médicos diretos cobertos no âmbito das operadoras de planos de saúde, isto é, para custear as duas alternativas em análise apenas os custos de honorários profissionais e recursos diretamente relacionados à assistência à saúde foram considerados. Foram usados os dados obtidos em ensaio clínico e avaliação de custo-efetividade anteriores 19,20; para complementar a construção da estimativa de custos, informações detalhadas serão descritas na próxima seção. Dado o curto prazo para a identificação do desfecho de interesse, foi adotado horizonte de tempo de 1 ano, não sendo aplicável, neste caso, taxa de desconto.

\section{Dados de entrada}

Em relação aos parâmetros clínicos para a construção do modelo (probabilidade do desfecho de interesse), foi utilizada a redução na taxa de parto prematuro, em específico os resultados para a análise de subgrupo para gestantes de baixo risco. A revisão sistemática identificou, para esse desfecho, um $\mathrm{RR}=0,71$ (IC95\%: 0,54; 0,92, 5 estudos, 9.726 gestantes de baixo risco; $\mathrm{p}=0,011$ ). Convém ressaltar que a metanálise de Sandall et al. ${ }^{1}$ comparou modelos de cuidado com enfermeiras obstetras e/ou obstetrizes que incluíam não apenas o pré-natal, mas também o parto e o pós-parto em alguns estudos. Optou-se por selecionar apenas o desfecho de parto prematuro, uma vez que está mais diretamente 
Diagrama da árvore de decisão comparando acompanhamento pré-natal por enfermeiros obstetras e/ou obstetrizes versus médicos de família ou obstetras.

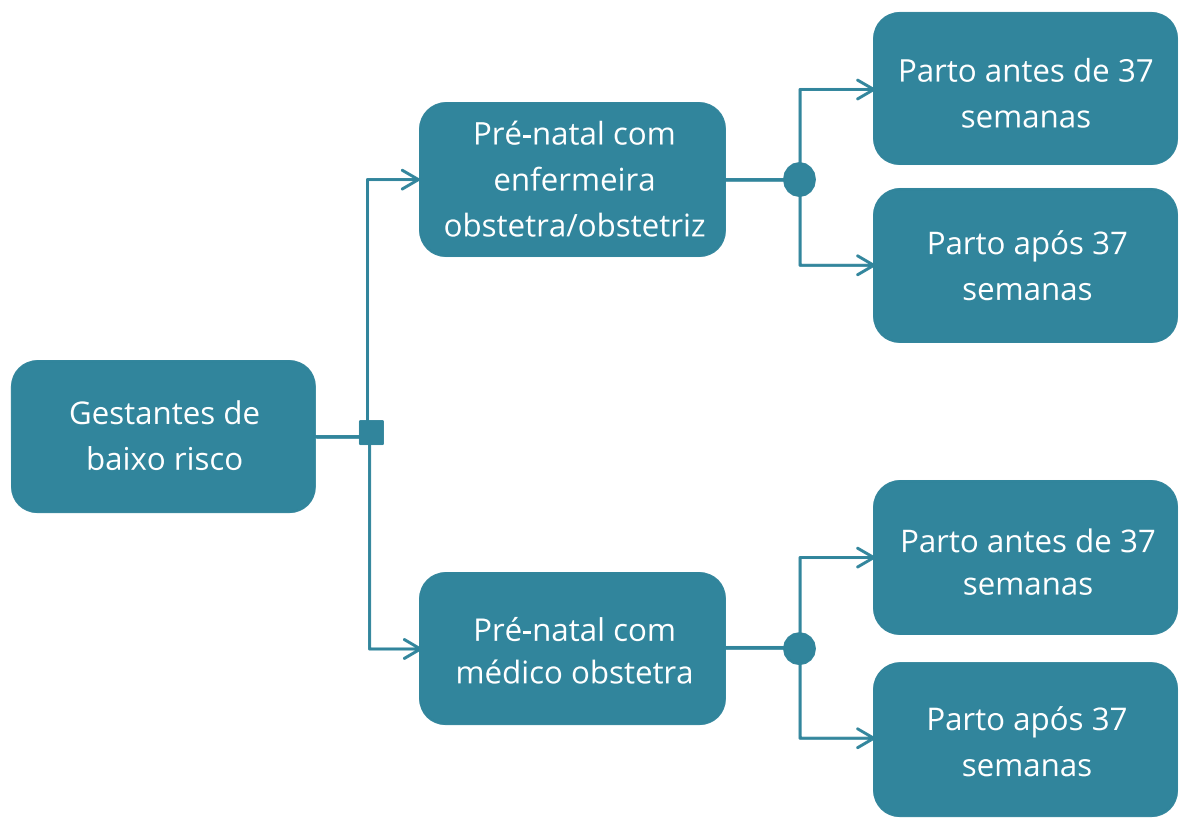

relacionado à assistência pré-natal. Essa premissa foi validada por um painel de especialistas composto por cinco profissionais médicos obstetras, duas profissionais enfermeiras obstetras ou obstetrizes e uma pediatra neonatologista, tendo encontrado respaldo clínico e científico considerado aceitável pelo painel.

Considerou-se que a taxa de parto prematuro em gestantes de baixo risco no Brasil, no Sistema de Saúde Suplementar, seria compatível com aquela observada em Sandall et al. ${ }^{1}$ no grupo do modelo de cuidado centrado no médico (5,44\%). É provável que essa taxa seja superior, mesmo em gestantes de baixo risco, uma vez que a taxa global reportada pelo estudo Nascer no Brasil foi de 11,5\%, considerando serviços públicos e privados e gestantes de risco variável. Para não superestimar o efeito da intervenção, adotou-se para os fins desta análise um risco de parto prematuro de 5,44\% no grupo médicos de família ou obstetras e uma redução para 4,10\% com a adoção do pré-natal com enfermeiras obstetras e/ou obstetrizes.

Para fins de definição dos custos associados a cada tecnologia, calculou-se o custo estimado com o acompanhamento pré-natal com enfermeiras obstetras e/ou obstetrizes, utilizando-se o número mínimo de consultas estabelecido pelo Manual Técnico do Ministério da Saúde e os exames previstos pelo Caderno de Atenção Básica 32 16, que estabelece as rotinas do Ministério da Saúde para o pré-natal de risco habitual, conforme descrito na Tabela 1. Para definir os custos associados ao pré-natal acompanhado por médicos de família ou obstetras, foi utilizado o percentual de aumento nos custos de pré-natais quando gestantes de risco habitual foram acompanhadas por médicos de família ou obstetras (por comparação ao cuidado com enfermeiras obstetras e/ou obstetrizes) reportado no estudo de Ratcliffe et al. 20. O estudo foi uma avaliação de custo-efetividade aninhada ao ensaio clínico randomizado de Tucker et al. 19, que comparou os desfechos clínicos do pré-natal com parteiras profissionais versus médicos obstetras na Escócia, com base nos dados de custos médicos diretos de 1.667 gestantes de risco habitual. Os autores observaram um aumento de custos com a adoção do pré-natal com médi- 
Tabela 1

Dados de custo unitário utilizados no modelo.

\begin{tabular}{|c|c|c|c|c|c|c|}
\hline Descrição & Valor (R\$) & Fonte & $\%$ uso & $\mathbf{n}$ & Fonte & $\begin{array}{l}\text { Valor utilizado } \\
\text { no modelo ( } \$ \text { \$) }\end{array}$ \\
\hline $\begin{array}{l}\text { Consulta pré-natal com enfermeiras obstétricas e/ou } \\
\text { obstetrizes }\end{array}$ & 65,00 & $\begin{array}{l}\text { Planserv-BA } \\
2016\end{array}$ & 100,00 & 6 & $\begin{array}{l}\text { Manual Técnico } \\
\text { Ministério da Saúde } 15\end{array}$ & 390,00 \\
\hline Consulta pré-natal com médicos de família e obstetras & 65,00 & $\begin{array}{l}\text { Planserv-BA } \\
2016\end{array}$ & 100,00 & 6 & $\begin{array}{l}\text { Manual Técnico } \\
\text { Ministério da Saúde } 15\end{array}$ & 390,00 \\
\hline \multicolumn{7}{|l|}{$\begin{array}{l}\text { Custos com exames e procedimentos aplicáveis ao } \\
\text { grupo do pré-natal com obstétricas e/ou obstetrizes }\end{array}$} \\
\hline Hemograma & 18,01 & CBHPM & 100,00 & 3 & $C A B, 201216$ & 54,03 \\
\hline Tipagem sanguínea & 26,73 & CBHPM & 100,00 & 1 & CAB, 201216 & 26,73 \\
\hline Coombs indireto & 26,73 & CBHPM & 9,66 & 3 & Baiochi et al. 33 * & 7,75 \\
\hline EAS/Urina I & 17,37 & CBHPM & 100,00 & 2 & CAB, 201216 & 34,74 \\
\hline Urocultura & 37,64 & CBHPM & 100,00 & 2 & CAB, 201216 & 75,28 \\
\hline Glicemia de jejum & 8,12 & CBHPM & 100,00 & 1 & CAB, 201216 & 8,12 \\
\hline TTOG 75g & 11,85 & CBHPM & 100,00 & 1 & $C A B, 201216$ & 11,85 \\
\hline Toxoplasmose IgG & 37,04 & CBHPM & 100,00 & 1 & CAB, 201216 & 37,04 \\
\hline Toxoplasmose IgM & 44,97 & CBHPM & 100,00 & 1 & CAB, 201216 & 44,97 \\
\hline VDRL & 15,53 & CBHPM & 100,00 & 2 & $C A B, 201216$ & 31,06 \\
\hline HbSAg & 54,02 & CBHPM & 100,00 & 2 & CAB, 201216 & 108,04 \\
\hline HIV & 60,20 & CBHPM & 100,00 & 2 & $C A B, 201216$ & 120,40 \\
\hline Ultrassonografia obstétrica 10 trimestre & 397,76 & CBHPM & 100,00 & 1 & Premissa & 397,76 \\
\hline Ultrassonografia morfológica $1 \underline{\text { o trimestre }}$ & 317,80 & CBHPM & 100,00 & 1 & Premissa & 317,80 \\
\hline Ultrassonografia morfológica 2o trimestre & 420,78 & CBHPM & 100,00 & 1 & Premissa & 420,78 \\
\hline Total & - & - & 100,00 & 1 & - & $1.696,34$ \\
\hline
\end{tabular}

CBHPM: Classificação Brasileira Hierarquizada de Procedimentos em Saúde; Planserv-BA: Sistema de Assistência à Saúde dos Servidores Públicos Estaduais do governo da Bahia; TTOG 75g: teste de tolerância oral à glicose de 75g.

Fonte: Associação Médica Brasileira (CBHPM. https://amb.org.br/cbhpm/, acessado em 4/Jan/2020).

* Fonte para o \% de gestantes Rh negativo no país.

co especialista quando comparado ao cuidado com enfermeiras obstetras e/ou obstetrizes de 7,89\% (de GBP 450,19 para GBP 417,28), derivado do aumento de custos com exames e procedimentos, mas também do custo global do cuidado. Esse aumento de 7,89\% foi aplicado aos custos calculados com o acompanhamento pré-natal com enfermeiras obstetras e/ou obstetrizes, apresentado na Tabela 1.

Em termos dos custos unitários, o valor da consulta foi considerado o mesmo daquele pago ao médico obstetra no modelo. Essa abordagem foi usada por ser conservadora e atender a demandas históricas das diversas profissões de saúde por isonomia de remuneração para procedimentos compatíveis. O valor usado foi de R $\$ 65,00$, utilizando como referência o valor da tabela de procedimentos do Sistema de Assistência à Saúde dos Servidores Públicos Estaduais do governo da Bahia (Planserv-BA), operadora do tipo autogestão. Essa fonte foi utilizada uma vez que a instituição publica em sua página os valores referenciais para pacotes de procedimentos, incluindo métodos para o cálculo do valor, de modo a permitir transparência dos valores praticados (Planserv Bahia. Valores referenciais. http://www.planserv.ba.gov.br/prestador/valores-referenciais/; Tabelas Planserv. http://www.plan serv.ba.gov.br/prestador/tabelas-planserv/, acessado em 30/Abr/2019). Desse modo, o valor final estimado para o acompanhamento pré-natal por enfermeiras obstetras e/ou obstetrizes, durante toda a gestação, foi de $\mathrm{R} \$ 2.086,34$, resultante da soma do custo com consultas e dos exames necessários ao seguimento de risco habitual. Para estimar o custo total do acompanhamento pré-natal com médicos de família ou obstetras, aplicou-se o aumento de 7,89\% observado em Ratcliffe et al. 20 sobre o custo de acompanhamento com enfermeiras obstetras e/ou obstetrizes, resultando em um total por gestação de $\mathrm{R} \$ 2.220,18$. 
Nessa estimativa de custos não foram contempladas as reduções nos valores de hospitalização e manejo desses partos prematuros, incluindo hospitalizações em unidade de terapia intensiva (UTI) neonatal, uma vez que a redução de partos prematuros é contemplada como desfecho de efetividade no modelo, evitando dupla contagem de benefícios 21 .

Foi conduzida ainda uma análise de sensibilidade univariada, para estimar cenários alternativos para os dados de custos unitários, conceitualmente mais sujeitos à variação a depender das tabelas praticadas pelas operadoras de planos de saúde ou a realidades locais diversas. Para isso, duas variáveis foram utilizadas na análise de sensibilidade: aumento percentual dos custos globais de acompanhamento pré-natal quando o modelo centrado no médicos de família ou obstetras é adotado (caso base $7,89 \%$ conforme Ratcliffe et al. 20 , variação de $2 \%$ a 25\%) e custo final do pré-natal exceto consultas (caso base R\$ 1.696,34, variação de $-50 \%$ até $+100 \%$ ou de $\mathrm{R} \$$ 848,17 até $\mathrm{R} \$ 3,392.69$ ).

\section{Resultados}

Utilizando-se os métodos apresentados anteriormente, bem como os dados de entrada descritos, calculou-se o custo incremental associado à substituição do pré-natal com médicos de família ou obstetras pelo modelo de cuidado pré-natal com enfermeiras obstetras e/ou obstetrizes, bem como o número de partos prematuros evitados a cada mil gestantes. Os cálculos da árvore de decisão, assim como a análise por mil gestantes, estão apresentados na Tabela 2.

Desse modo, calculou-se o custo incremental, a efetividade incremental e a RCEI, conforme apresentado na Tabela 3. Como é possível observar, o pré-natal com enfermeiras obstetras e/ou obstetrizes mostrou-se uma opção dominante, isto é, que resulta em maior efetividade e menores custos. Esse resultado deve-se à redução dos custos com assistência pré-natal de gestantes de risco habitual e, simultaneamente, à prevenção de partos prematuros. A RCEI estimada foi de -R \$ 10.038,43 por parto prematuro evitado (uma economia de $\mathrm{R} \$ 10.038,43$ a cada parto prematuro evitado).

Tabela 2

Estimativa de cálculos da árvore de decisão e análise por 1.000 gestantes.

\begin{tabular}{|c|c|c|c|}
\hline Comparador/Braços & Probabilidade do desfecho & $\begin{array}{l}\text { Custos unitários de } \\
\text { acompanhamento }\end{array}$ & Custos do braço * \\
\hline \multicolumn{4}{|l|}{$\begin{array}{l}\text { Pré-natal com enfermeiras obstétricas e/ou } \\
\text { obstetrizes }\end{array}$} \\
\hline Com parto prematuro & $4,10 \%$ & $\mathrm{R} \$ 2.086,34$ & $\mathrm{R} \$ 85,60$ \\
\hline Sem parto prematuro & $95,90 \%$ & $\mathrm{R} \$ 2.086,34$ & $\mathrm{R} \$ 2.000,74$ \\
\hline \multicolumn{4}{|l|}{ Pré-natal com médicos de família ou obstetras } \\
\hline Com parto prematuro & $5,44 \%$ & $\mathrm{R} \$ 2.220,18$ & $\mathrm{R} \$ 120,69$ \\
\hline Sem parto prematuro & $94,56 \%$ & $\mathrm{R} \$ 2.220,18$ & $\mathrm{R} \$ 2.099,49$ \\
\hline \multicolumn{4}{|l|}{ Análise por 1.000 gestantes } \\
\hline \multicolumn{4}{|c|}{ Pré-natal com enfermeiras obstétricas e/ou obstetrizes } \\
\hline Número de gestantes com parto prematuro & & & 41 \\
\hline Número de gestantes sem parto prematuro & & & 959 \\
\hline Custos com gestantes com parto prematuro & & & $\mathrm{R} \$ 85.599,22$ \\
\hline Custos com gestantes sem parto prematuro & & & $\mathrm{R} \$ 2.000 .744,03$ \\
\hline \multicolumn{4}{|l|}{ Pré-natal com médicos de família ou obstetras } \\
\hline Número de gestantes com parto prematuro & & & 54 \\
\hline Número de gestantes sem parto prematuro & & & 945 \\
\hline Custos com gestantes com parto prematuro & & & $\mathrm{R} \$ 120.692,03$ \\
\hline Custos com gestantes sem parto prematuro & & & $\mathrm{R} \$ 2.099 .492,71$ \\
\hline
\end{tabular}

* Os custos do braço representam a multiplicação da probabilidade da ocorrência daquele braço/desfecho pelo custo de acompanhamento correspondente àquele braço. 
Resultados da avaliação de custo-efetividade.

\begin{tabular}{lccc}
\hline & $\begin{array}{c}\text { Pré-natal com enfermeiras } \\
\text { obstetras e/ou obstetrizes }\end{array}$ & $\begin{array}{c}\text { Pré-natal com médicos de } \\
\text { família ou obstetras }\end{array}$ & Incremental \\
\hline $\begin{array}{l}\text { Custo total } \\
\text { Número de pacientes com parto prematuro }\end{array}$ & $\mathrm{R} \$ 2.086 .343,25$ & $\mathrm{R} \$ 2.220 .184,74$ & $-\mathrm{R} \$ 133.841,48$ \\
$\begin{array}{l}\text { (<37 semanas }) \\
\text { Razão de custo-efetividade incremental }\end{array}$ & 41 & 54 \\
\hline
\end{tabular}

A Figura 2 apresenta os resultados para cada uma das análises de sensibilidade univariada para as variáveis aumento percentual dos custos globais de pré-natal com médicos de família ou obstetras, quando comparados a enfermeiras obstetras e/ou obstetrizes, e custo de acompanhamento pré-natal exceto consultas, respectivamente. Em todos os cenários simulados, a RCEI permanece indicando um cenário dominante em favor do acompanhamento pré-natal por enfermeiras obstetras e/ou obstetrizes, com economias associadas à substituição variando de - $\mathrm{R} \$ 2.544,60$ até $-\mathrm{R} \$ 31.807,46$.

\section{Discussão}

Em termos econômicos, com base nos dados de entrada e do modelo de decisão construído, estimouse uma economia de $-\mathrm{R} \$ 10.038,43$ associada à redução de um caso de parto prematuro $(<37$ semanas), mostrando que a tecnologia de cuidado pré-natal por enfermeiras obstetras e/ou obstetrizes é dominante (tem maior efetividade e menor custo), comparada ao cuidado com médicos médicos de família ou obstetras, no cenário clínico da gestação de risco habitual e na perspectiva da operadora de plano de saúde como fonte pagadora. Desse modo, a cada 1.000 gestantes de baixo risco acompanhadas durante o pré-natal por enfermeiras obstetras e/ou obstetrizes, estima-se uma redução de 13 casos de parto prematuro no âmbito do Sistema de Saúde Suplementar e uma economia de recursos da ordem de R $\$ 133.841,48$, associada em particular à redução dos custos globais de pré-natal. Esses resultados foram testados na análise de sensibilidade univariada, mostrando consistência da economia de recursos observada para todas as faixas de variação testadas. Conforme foi possível observar, em todos os cenários testados a RCEI mantém-se negativa, isto é, o pré-natal com enfermeiras obstetras e/ou obstetrizes é poupador de recursos mesmo quando resulta em alterações mínimas no custo global de pré-natal (pela redução no consumo de exames e procedimentos desnecessários no baixo risco) ou quando são testados diferentes custos globais de acompanhamento pré-natal. Esse resultado é observado uma vez que o pré-natal com enfermeiras obstetras e/ou obstetrizes é uma tecnologia que substitui uma já existente, sem onerar os custos totais do sistema, e ainda impactando positivamente nos desfechos clínicos de interesse.

A assistência pré-natal oferecida por enfermeiras obstetras e obstetrizes para gestantes de risco habitual é amplamente recomendada em diretrizes nacionais e internacionais, incluindo documentos do Ministério da Saúde brasileiro 3,4,5,15,16,17. De acordo com as evidências de alta qualidade metodológica, além da melhora de desfechos maternos e perinatais 1,22, o cuidado realizado por enfermeiras obstetras e/ou obstetrizes pode resultar em impacto econômico significativo 19,20, quando comparado ao acompanhamento realizado predominantemente por médicos ginecologistas e obstetras. Nossos achados parecem indicar que tais benefícios seriam relevantes no cenário brasileiro do Sistema de Saúde Suplementar, tendo em vista a redução consistente dos custos associados ao acompanhamento pré-natal por enfermeiras obstetras e/ou obstetrizes em todos os cenários analisados.

Entende-se que esses benefícios poderiam ser ainda maiores em contextos e circunstâncias específicos. Por exemplo, Villar et al. 14 observaram que não houve diferença para a taxa de cesárea entre os grupos acompanhados por enfermeiras obstetras e/ou obstetrizes e aqueles acompanhados por 
Figura 2

Análise de sensibilidade univariada.

2a) Percentual de aumento dos custos de pré-natal com médicos de família ou obstetras

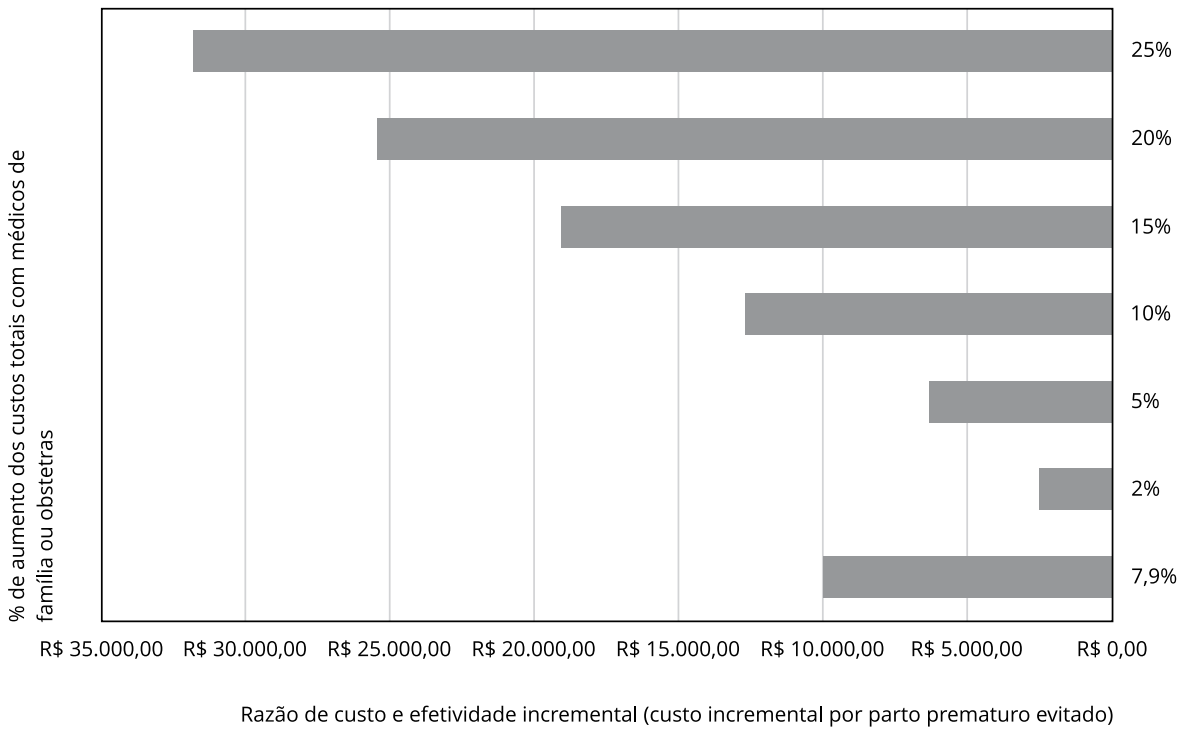

2b) Custos de pré-natal exceto consultas

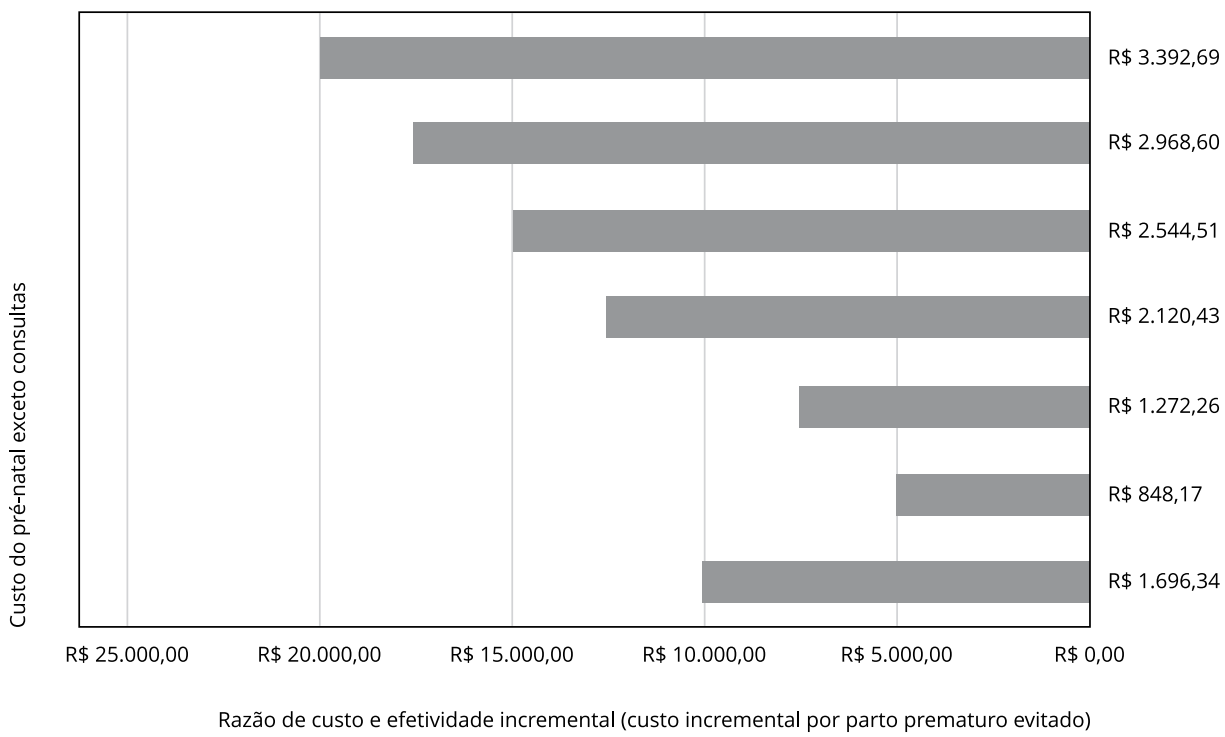

médicos de família ou obstetras durante o pré-natal, no entanto, a taxa de cesárea nos ensaios clínicos randomizados foi cerca de $11 \%$ em ambos os grupos, taxa muito difícil de ser reduzida, mesmo no cenário clínico do risco habitual. No contexto do Sistema de Saúde Suplementar brasileiro, as taxas de cesárea são da ordem de $80-90 \%$ 23, de modo que é possível acreditar que a incorporação do pré-natal com enfermeiras obstetras e/ou obstetrizes neste cenário poderia gerar impactos mais pronunciados sobre tal desfecho. Considerando as alarmantes taxas de cesárea no país e seu reconhecimento como 
um problema de saúde pública 24,25, esta provavelmente seja uma tecnologia com potencial para a reduzir cesáreas e intervenções obstétricas, uma das metas para que o país alcance melhores parâmetros de saúde materno-infantil 1,26.

Considera-se, ainda, que essas economias poderiam ser mais significativas caso fosse contemplada também a redução de custos com a admissão neonatal e o manejo da prematuridade, bem como de complicações de longo prazo dos nascimentos prematuros 11,27. Adicionalmente, se estudos conduzidos em cenário nacional vierem a demonstrar redução na taxa de cesárea associada ao pré-natal com enfermeiras obstetras e/ou obstetrizes, por exemplo, pela realização de atividades educativas pré-natais com vistas à escolha informada sobre a via de nascimento, esses benefícios econômicos poderiam ser ainda mais significativos para o sistema, impactando não apenas na redução de custos com a cirurgia, mas também do manejo de complicações mais frequentes após cesáreas (histerectomia, internação em UTI materna, transfusão sanguínea, infecção e outras) 5,7,28,29,30.

A presente análise de custo-efetividade foi conduzida utilizando-se metodologia habitual em estudos econômicos 19 , tendo adotado abordagem conservadora no custeio dos recursos em saúde, bem como na seleção de desfechos. Adicionalmente, os dados clínicos usados no modelo para a estimativa de eficácia foram obtidos com base em uma revisão sistemática com metanálise de alta qualidade metodológica, publicada pela Colaboração Cochrane 1 .

No entanto, esta análise apresenta algumas limitações. A indisponibilidade de dados de mundo real para o custeio das duas alternativas em análise pode ter comprometido a importância das estimativas de custo, que foram baseadas unicamente em protocolos e na validação com painel de especialistas. Estudos observacionais prospectivos ou retrospectivos do tipo custo da doença poderiam informar de modo mais abrangente os custos de acompanhamento com cada comparador. Adicionalmente, o aumento percentual de custo associado ao acompanhamento com médicos de família ou obstetras foi obtido baseando-se em um estudo realizado em outro país e há mais de 20 anos (Ratcliffe et al. 20), pela indisponibilidade de dados similares mais atuais e no contexto nacional. Não é possível prever se tais limitações podem ter superestimado ou subestimado os resultados finais em favor do acompanhamento pré-natal com enfermeiras obstetras e/ou obstetrizes. No entanto, parece seguro afirmar que a quantidade de exames complementares vem aumentando ao longo dos anos, muitos destes sem justificativa clínica 31,32 . Outro ponto relevante é que não é possível descartar que o conteúdo das consultas pré-natais por enfermeiras obstetras e/ou obstetrizes, bem como cointervenções disponíveis no sistema de saúde dos países avaliados na metanálise de Sandall et al. 1, utilizada para obter os parâmetros clínicos do modelo, sejam diferentes da realidade local no Brasil.

Como conclusão, as evidências apontam resultados maternos e neonatais comparáveis (em termos de complicações) para a assistência pré-natal prestada por enfermeiras obstetras e obstetrizes quando comparada àquela prestada por médicos obstetras, com superioridade do modelo de pré-natal prestado por enfermeiras e obstetrizes para o desfecho de prevenção de partos prematuros. Esse benefício é particularmente importante considerando-se a elevada taxa de partos prematuros no país, particularmente no âmbito do Sistema de Saúde Suplementar. Esses benefícios clínicos são acompanhados ainda de uma estimativa de economias significativas para o sistema de saúde, em todos os cenários analisados. 


\section{Colaboradores}

M. O. Menezes e M. L. S. Takemoto participaram da concepção e planejamento do estudo, bem como da coleta e análise de dados, redação da versão inicial do manuscrito e revisão e aprovação da versão final. R. Knobel e C. B. Andreucci participaram da concepção e planejamento do estudo, análise de dados, redação da versão inicial do manuscrito e revisão e aprovação da versão final. C. G. Magalhães, M. M. R. Amorim e L. Katz participaram da concepção e planejamento do estudo, análise de dados e aprovação da versão final.

\section{Informações adicionais}

ORCID: Mariane de Oliveira Menezes (00000002-8525-0521); Roxana Knobel (0000-00019180-4685); Carla Betina Andreucci (0000-00025590-108X); Claudia Garcia Magalhães (00000001-7033-1807); Melania Maria Ramos Amorim (0000-0003-1047-2514); Leila Katz (0000-00019854-7917); Maíra Libertad Soligo Takemoto (0000-0002-7016-2879).

\section{Agradecimentos}

Os autores agradecem a todas as pessoas (profissionais da perinatalidade, ativistas do parto, doulas, gestantes e famílias) que contribuíram com a campanha de financiamento coletivo que permitiu a realização deste estudo. Agradecem ainda aos especialistas que validaram a hipótese adotada no modelo.

\section{Referências}

1. Sandall J, Soltani H, Gates S, Shennan A, Devane D. Midwife-led continuity models versus other models of care for childbearing women. Cochrane Database Syst Rev 2016; 4:CD004667.

2. Brasil. Lei no 7.498, de 25 de Junho de 1986. Dispõe sobre a regulamentação do exercício da enfermagem, e dá outras providências. Diário Oficial da União 1986; 26 jun.

3. International Confederation of Midwives. Essential competencies for midwifery practice. https://www.internationalmidwives.org/as sets/files/general-files/2019/03/icm-com petencies-en-screens.pdf (acessado em 29/ Abr/201).

4. Organização Mundial da Saúde. Recomendações da OMS sobre cuidados pré-natais para uma experiência positiva na gravidez. https://apps.who.int/iris/bitstream/hand le/10665/250800/WHO-RHR-16.12-por.pdf? sequence $=2$ (acessado em 29/Abr/2019).

5. World Health Organization. WHO recommendations non-clinical interventions to reduce unnecessary caesarean sections. https://apps.who.int/iris/bitstream/handle/ 10665/275377/9789241550338-eng.pdf?ua =1 (acessado em 29/Abr/2019).

6. Souza J, Gülmezoglu A, Lumbiganon P, Laopaiboon M, Carroli G, Fawole B, et al. Caesarean section without medical indications is associated with an increased risk of adverse short-term maternal outcomes: the 2004-2008 WHO Global Survey on Maternal and Perinatal Health. BMC Med 2010; 8:71.

7. Domingues RMSM, Dias MAB, Schilithz AOC, Leal MC. Factors associated with maternal near miss in childbirth and the postpartum period: findings from the birth in Brazil National Survey, 2011-2012. Reprod Health 2016; 13 Suppl 3:115.

8. Batista Filho M, Rissin A, Batista Filho M, Ris$\sin \mathrm{A}$. WHO and the epidemic of cesarians. Rev Bras Saúde Matern Infant 2018; 18:3-4.

9. World Health Organization. WHO statement on caesarean section rates. https://apps.who. int/iris/bitstream/handle/10665/161442/ WHO_RHR_15.02_eng.pdf;jsessionid=7E33C 437CDBD26C8DC205B5779D015ED?sequen $\mathrm{ce}=1$ (acessado em 02/Mai/2019).

10. World Health Organization. Strategies toward ending preventable maternal mortality (EPMM). https://apps.who.int/iris/bitstream/ handle/10665/153544/9789241508483_eng. pdf;jsessionid=7686E3D0C7B6E63F536914 CDD8FC797E? sequence $=1$ (acessado em 30/ $\mathrm{Abr} / 2019)$.

11. Leal MC, Esteves-Pereira AP, Nakamura-Pereira $M$, Torres JA, Theme-Filha $M$, Domingues RMSM, et al. Prevalence and risk factors related to preterm birth in Brazil. Reprod Health 2016; 13 Suppl 3:127. 
12. Tedesco RP, Passini R Jr, Cecatti JG, Camargo RS, Pacagnella RC, Sousa MH. Estimation of preterm birth rate, associated factors and maternal morbidity from a demographic and health survey in Brazil. Matern Child Health J 2013; 17:1638-47.

13. Oliveira RR, Melo EC, Fujimori E, Mathias TAF. The inner state differences of preterm birth rates in Brazil: a time series study. BMC Public Health 2016; 16:411.

14. Villar J, Carroli G, Khan-Neelofur D, Piaggio GG, Gülmezoglu AM. Patterns of routine antenatal care for low-risk pregnancy. Cochrane Database Syst Rev 2001; (4):CD000934.

15. Soares Filho AM, Serra ASL, Cruz DRN, Pires HMB, Schirmer J, Drezett J, et al. Pré-natal e puerpério atenção qualificada e humanizada manual técnico. Brasília: Ministério da Saúde; 2005. (Série A. Normas e Manuais Técnicos) (Série Direitos Sexuais e Direitos Reprodutivos - Caderno 5).

16. Departamento de Atenção Básica, Secretaria de Atenção à Saúde, Ministério da Saúde. Cadernos de atenção ao pré-natal de baixo risco. Brasília: Ministério da Saúde; 2012. (Série A. Normas e Manuais Técnicos) (Cadernos de Atenção Básica, 32).

17. National Institute for Health and Care Excellence. Antenatal care for uncomplicated pregnancies. https://www.nice.org.uk/guidance/ cg62/resources/antenatal-care-for-uncompli cated-pregnancies-pdf-975564597445 (acessado em 29/Abr/2019).

18. Departamento de Ciência e Tecnologia, Secretaria de Ciência, Tecnologia e Insumos Estratégicos, Ministério da Saúde. Diretrizes metodológicas: diretriz de avaliação econômica. Brasília: Ministério da Saúde; 2014.

19. Tucker JS, Hall MH, Howie PW, Reid ME, Barbour RS, du Florey CV, et al. Should obstetricians see women with normal pregnancies? A multicentre randomised controlled trial ofroutine antenatal care by general practitioners and midwives compared with shared care led by obstetricians. https://europepmc.org/ backend/ptpmcrender.fcgi?accid=PMC23503 48\&blobtype $=$ pdf (acessado em 29/Abr/2019).

20. Ratcliffe J, Ryan M, Tucker J. The costs of alternative types of routine antenatal care for low-risk women: shared cTare vs care by general practitioners and community midwives. J Health Serv Res Policy 1996; 1:135-40.

21. Drummond MF, Sculpher MJ, Torrance GW, O'Brien BJ, Stoddart GL. Methods for the economic evaluation of health care programmes. 2nd Ed. Oxford: Oxford University Press; 1997.

22. Villar J, Carroli G, Khan-Neelofur D, Piaggio GG, Gulmezoglu M, Gülmezoglu AM, et al. Patterns of routine antenatal care for low-risk pregnancy (review). Cochrane Database Syst Rev 2001; (4):CD000934.
23. Domingues RMSM, Dias MAB, Nakamura -Pereira M, Torres JA, D’Orsi E, Pereira APE, et al. Processo de decisão pelo tipo de parto no Brasil : da preferência inicial das mulheres à via de parto final. Cad Saúde Pública 2014; 30 Suppl:S101-16.

24. Leal MC, Pereira APE, Domingues RMSM, Theme Filha MM, Dias MAB, Nakamura-Pereira $\mathrm{M}$, et al. Intervenções obstétricas durante o trabalho de parto e parto em mulheres brasileiras de risco habitual. Cad Saúde Pública 2014; 30 Suppl:S17-47.

25. Salgado HO, Queiroz MR, Santos HG, Andreucci CB, Diniz CSG. Using the maternity safety thermometer to estimate harm-free care in Southeast Brazil: a hospital-based cohort. Birth 2019; 46:583-91.

26. McLachlan HL, Forster DA, Davey MA, Farrell T, Gold L, Biro MA, et al. Effects of continuity of care by a primary midwife (caseload midwifery) on caesarean section rates in women of low obstetric risk: the COSMOS randomised controlled trial. BJOG 2012; 119:1483-92.

27. Leal MC, Esteves-Pereira AP, NakamuraPereira M, Domingues RMSM, Dias MAB, Moreira ME, et al. Burden of early-term birth on adverse infant outcomes: a populationbased cohort study in Brazil. BMJ Open 2017; 7:e017789.

28. Entringer AP, Pinto M, Dias MAB, Gomes MASM. Análise de custo-efetividade do parto vaginal espontâneo e da cesariana eletiva para gestantes de risco habitual no Sistema Único de Saúde. Cad Saúde Pública 2018; 34:e00022517.

29. Nakamura-Pereira M, Leal MC, EstevesPereira AP, Domingues RMSM, Torres JA, Dias MAB, et al. Use of Robson classification to assess cesarean section rate in Brazil: the role of source of payment for childbirth. Reprod Health 2016; 13 Suppl 3:128.

30. Souza J, Gulmezoglu AM, Lumbiganon P, Laopaiboon M, Carrin G, Fawole B, et al. Caesarean section without medical indications is associated with an increased risk of adverse short-term maternal outcomes: the 2004-2008 WHO Global Survey on Maternal and Perinatal Health. BMC Med 2010; 8:71.

31. Amorim MMR, Melo ASO. Avaliação dos exames de rotina no pré-natal - parte 1. Rev Bras Ginecol Obstet 2009; 31:148-55.

32. Amorim MMR, Melo ASO. Avaliação dos exames de rotina no pré-natal - parte 2. Rev Bras Ginecol Obstet 2009; 31:367-74.

33. Baiochi E, Camano L, Sass N, Colas OR. Freqüências dos grupos sanguíneos e incompatibilidades $\mathrm{ABO}$ e $\mathrm{RhD}$ em puérperas e seus recém-nascidos. Rev Assoc Med Bras (1992) 2007; 53:44-6. 
Abstract

In several countries, primary care for pregnant women is performed by obstetric nurses and/or midwives. In Brazil's Supplementary Health System (private health insurance and out-of-pocket care), coverage of prenatal care is mandatory and is performed by medical obstetricians. The objective of this study is to conduct a cost-effectiveness analysis, comparing clinical outcomes and costs associated with the incorporation of prenatal care by obstetric nurses and midwives in the Supplementary Health System, from the perspective of the operator of health plans as the payment source. A decision tree was built, based on data from a Cochrane Collaboration meta-analysis that showed a reduction in the risk of premature birth in the group of normal-risk pregnant women accompanied by obstetric nurses and midwives. The analysis only considered the direct medical costs covered by health plan operators for essential appointments and tests, according to the prevailing Ministry of Health protocol. The study assumed equal unit costs of consultations by medical professionals and applied an increase in the overall cost of prenatal tests associated with medical follow-up, based on data from the literature. Incremental cost-effective ratio was estimated at $-B R L$ 10,038.43 (savings of BRL 10,038.43) per premature birth avoided. This result was consistent with the sensitivity analyses, with savings associated with the substitution ranging from - BRL 2,544.60 to -BRL 31,807.46 per premature death avoided. In conclusion, prenatal care provided by obstetric nurses and midwives was superior to that provided by medical obstetricians for the prevention of premature birth, besides resulting in cost savings.

Health Expenditures; Cost-Benefit Analysis; Prenatal Care; Obstetrics; Health Promotion

\section{Resumen}

En diversos países, la atención primaria a las gestantes se realiza con enfermeras obstetras $y / o$ parteras. En el Sistema Suplementario de Salud en Brasil, la cobertura de la asistencia prenatal es obligatoria y la realizan médicos obstetras. El objetivo de este estudio es realizar un análisis de costo-efectividad, comparando resultados clínicos $y$ costes asociados a la incorporación en el periodo prenatal de enfermeras obstetras y parteras, en el ámbito del Sistema de Salud Suplementaria, desde la perspectiva de una operadora de planes de salud como fuente pagadora. Se construyó un árbol de decisión, basado en datos de metaanálisis de la Colaboración Cochrane, que mostró una reducción del riesgo de parto prematuro en el grupo de gestantes de riesgo habitual, con un seguimiento de enfermeras obstetras y parteras. Se consideraron solo los costes médicos directos, cubiertos por las operadoras de planes de salud para la realización de consultas y exámenes esenciales, conforme el protocolo vigente del Ministerio de Salud. Se asumió el coste unitario de consulta con cada profesional como iguales, y se aplicó un aumento del coste global con exámenes prenatales asociado al seguimiento médico, conforme los datos obtenidos en la literatura. Se estimó la razón de costo-efectividad incremental de -BRL 10.038,43 (economía de BRL 10.038,43) por parto prematuro evitado. Este resultado se mostró consistente en los análisis de sensibilidad, con ahorros asociados a la sustitución, variando de -BRL 2.544,60 hasta -BRL 31.807,46 por parto prematuro evitado. Como conclusión, se observó que el cuidado prenatal por parte de enfermeras obstetras y parteras es superior al prestado por médicos obstetras para el desenlace de prevención de parto prematuro, resultando incluso en un ahorro de recursos.

Gastos en Salud; Análisis Costo-Beneficio; Aténción Prenatal; Obstetricia; Promoción de la Salud
Recebido em 14/Abr/2020

Versão final reapresentada em 17/Nov/2020

Aprovado 17/Dez/2020 